\title{
A Radiance Model for Predicting Radio Wave Propagation in Irregular Dense Urban Areas
}

\author{
Eugenia Montiel ${ }^{1}$, Alberto Aguado $^{2}$, François Sillion $^{3}$ \\ 1. Centre for Communications and System Research \\ 2. Centre for Vision, Speech and Signal Processing, \\ University of Surrey, UK \\ 3. INRIA, iMAGIS-GRAVIR, France
}

\begin{abstract}
We present a deterministic model of radio wave propagation based on radiance transfers. Our model uses radiosity techniques to determine facet-to-facet specular reflections according to a 3D building description. The model contains two main components. First, visibility between elements is determined and used to establish links that represent radiance transfers that include diffraction and free space losses based on geometric approximations. Secondly, links are used to define a transfer equation whose solution provides the transfer intereflections. The solution is obtained by using hierarchical radiosity. The results of the model show a good agreement with measurements made in urban areas.
\end{abstract}




\section{Introduction}

The growth of wireless communications and the introduction of cellular communications to support a high density of users in highly dense urban areas have motivated a considerable interest in the simulation and prediction of radio wave propagation in urban environments. Path-loss can be computed by using empirical prediction models that include factors to account for the urbanisation, topology and antenna features [1][2][3]. Although these models are a valuable tool for estimating signal propagation, they generally produce significant errors for small covering ranges [4] (i.e., less than $1 \mathrm{~km}$ ), for low base station antennas and for irregular dense coverage areas. A major source of error is due to buildings of different size and irregular passageways. In these cases, deterministic models can produce more accurate path loss predictions [4][5][6][7][8][9][10][11].

Deterministic models use detailed 2D or 3D geometric description of the urban area to compute propagation [12][13]. A detailed description of the geometry makes possible to obtain a detailed path-loss prediction by modelling the power being transferred from a point to another. There are two main approaches to model power transferred. In the ray tracing approach, dominant paths determine points at which evaluate the intensity. Paths are computed by considering diffraction and reflection between elements such as building's facets and edges. Reflection and refraction rays are fired at each intersection from the path. This approach provides an accurate treatment of specular reflection and has been extensively exploited in radio wave propagation models. In a divergent approach, rather than fire rays from a point, it is possible to model the energy being transferred by considering all the ways in which a signal can reach a point. Thus, the intensity at all the points can be found by solving a system of equations that account for the reflection and absorption of all surfaces simultaneously. This approach is based on radiosity models derived from thermalengineering concepts [14] and it has been extensively used in the simulation of light [15]. The 
main advantage is that it provides an accurate treatment of inter object reflections. However, radiosity models have not been very popular in radio wave propagation [16][17][18]. The main drawback is that radiosity is expressed as a uniform dispersion, thus it does not model any specular component. It requires complementary models to account for diffuse reflections [18][19]. Additionally, radiosity models do not include diffraction and multipath fading. This paper develops a model that addresses these problems. Our model avoids the use of radiosity in favour of a development in terms of radiance that includes a model of the reflectance function. The reflectance function contains specular and diffuse components. Additionally, we model the iteration between facets by including diffraction and fading.

\section{Global Radiance Framework}

Figure 1 illustrates the main elements of our prediction model. We start from a 3D description of the elements in a specific geographic area. This description consists of a set of facets and edges that define buildings. Visibility between edges and facets is precomputed in two steps. The first step divides the 3D space into a set of elements in a regular grid. The second step uses this grid to determine the line-of-sight between edges and facets. The grid and visibility information is stored in the city database, thus it can be used for any alternative transmitter inputs.

The prediction starts by selecting a region in the 3D model based on the fourth-power distance [20]. This selection criteria produces an excess loss, thus it is only used to reduce computations by limiting the area of interest. In this area, we use the precomputed grid to determine the edges and facets that have a direct line-of-sight with the transmitter or the receiver. We select the elements that have a direct line-of-sight to the transmitter or the receiver and the elements that are visible from these elements. High order reflections are included by considering elements visible from the selected elements. After the elements that will contribute to the power propagation have been selected, we establish propagation links 
that indicate that the signal is propagated between facets. A link indicates that a facet transmits radio waves to other facets by means of reflection or diffraction. A facet can have many incoming and outcoming links forming a network in which each facet is considered as a multi-path receiver and transmitter. We use links to define a radiance equation that models the global signal transfers as a vector sum of many paths resulting from reflection and diffraction. Since the direct path from the receiver to the transmitter does not have a reflection or diffraction component, then the contribution for this path is not included in the radiance model. The transfer of this link is computed separately and then included in the final path loss prediction (see figure 1).

The global transfer solution is obtained by hierarchical radiosity techniques. Hierarchical radiosity is a powerful technique which computes all possible paths of radiant energy transfers at variable levels of resolution [15][21]. This technique subdivides facets into small surfaces forming a hierarchical structure. Thus, the interaction between two different root surfaces is represented as a number of links between different levels. The subdivision is important to obtain accurate transfers between finite small areas (accurate form factors and reflection points) and it accounts for partial occlusions. The solution is obtained by repetitively updating the energy transfers until the updates of the incident and emitted energies are small.

\section{Database}

Our database contains information about building geometry, antenna patterns and field measurements. The building database contains 303 buildings distributed over approximately an area of $1 \times 1 \mathrm{kms}$ in the Stuttgart region. Figure 2 shows a snapshot of the $3 \mathrm{D}$ building database. The database only provides geometric information and we do not have any reflectance properties of the buildings. 
We organised the building data following a hierarchical-geometric representation. A city is represented as a list of buildings. Each building is represented as a list of facets, each facet is composed of edges, each edge has two vertices, and a vertex is defined as threedimensional point with $(x, y, z)$ co-ordinates. Facets are defined as closed polygons that represent the roof and walls. Buildings are approximated by considering only vertical walls and horizontal roofs.

The database contains the position of two rooftop transmitters whose patterns are defined as the gain for the far-field region as a function of directional coordinates. For each transmitter, we know the absolute orientation of the antenna pattern. The antenna pattern is specified only at selected orientations in horizontal and vertical planes crossing at the emitter location. This data is interpolated to obtain the attenuation in any direction. Field measures in the $800 \mathrm{MHz}$ band are defined as a path of $2 \mathrm{D}$ positions with a height of $1.7 \mathrm{~m}$. Measures were obtained from two independent transmitters. Figure 2 shows the position of one transmitter and the corresponding receiver path.

\section{Visibility}

In order to compute propagation, we need to determine which building facets reflect and receive direct reflection and diffraction from other buildings. Reflection is produced when two facets have a direct line-of-sight, whilst diffraction is produced when an edge has a direct line-of-sight with two facets.

We compute visibility in two stages. First, we compute the visibility between the geometric elements in the 3D model. Since this visibility is independent of the location of the transmitter and the receivers, it can be precomputed and stored with the database. In a second step, we determine which geometrical elements have a direct line-of-sight with the receivers and with the transmitter. The visibility between two elements is determined by considering 
the visibility between two points. For a facet, if one of its corners is visible, then we consider that the facet is visible. For an edge, if one of its two end-points is visible, then we consider that the edge is visible. Additionally, edges in the same facet are considered visible to each other. It is important to notice that this visibility computation determines if a facet or part of it contributes to the path loss. This information is then used by the hierarchical algorithm to divide the facets in such a way that the visibility, the point of reflection and the actual area contribution (due to partial occlusions) is accurate. Thus, transfers occur between small areas of visible facets.

In order to determine visibility, we need to look for an intersection between the planes that define facets and the straight line that joins a pair of points. Given the large number of facets in a typical urban area, the computation of the visibility for all the elements in the model requires significant computational load. This is a demanding task even if visibility in the $3 \mathrm{D}$ model is precomputed. In order to reduce this complexity, we use a space volumetric partitioning technique. This technique is related to partitioning visibility algorithms [22][23], but instead of computing a graph that specify what can be seen from a certain point, we use the subdivision to search for intersections. That is, we reduce the number of intersection tests by reducing the number of facets to a predetermined region close to the tested line.

Figure 3 shows an example of the partition of the 3D space for a subset of buildings in the database. The partition divides the space into a set of cubes that define a 3D grid. The size of the grid is given by the average of the size of the buildings; thus, we expect to have one building by cube. Each cube contains a list to store the buildings that are intersected by its volume. Since the grid is independent of the location of the transmitter and the receivers, then it is precomputed and stored in the city database. The elements of the grid reduce computations by limiting the intersection tests to facets contained in the elements that define the trajectory of the straight line between two points. Figure 4 shows an example of the use of 
grid elements in the computation of visibility. Figure 4(a) shows a top view with the elements that define the trajectory between two points. Figure 4(b) shows a 3D view of the initial, final and the intersection obtained by the algorithm. Given two points $p_{i}$ and $p_{j}$, grid elements are incrementally computed by considering the trajectory of the line-of-sight given by

$$
\Gamma_{i, j}=p_{i}+q_{i, j} t
$$

where $q_{i, j}=\left(p_{j}-p_{i}\right) /\left\|p_{j}-p_{i}\right\|$ is the normalised vector defining the line's direction. The trajectory of the line defines a list of consecutive elements of the $3 \mathrm{D}$ grid. The first element in the list is the element of the grid that contains the point $p_{i}$. The last element in the list is the grid element that contains the point $p_{j}$. Give a grid element $e_{k}$, the next grid element $e_{k+1}$ in the list can be obtained by determining the intersection of the line in equation (1) with the six planes that form the facet of the element $e_{k}$. The element $e_{k+1}$ must be chosen in the direction of the plane that is first intersected by the line. This process is illustrated in Figure 5 . In this figure, we indicate in grey the planes that are intersected by the line $\Gamma_{i, j}$. The planes $\Omega_{1}$ and $\Omega_{2}$ are intersected one after the other. As such, the element $e_{k+1}$ is the grid element that shares the facet $\Omega_{2}$ with the current element $e_{k}$.

We represent planes by considering the homogeneous form

$$
\Omega^{T} p=0
$$

where $\Omega^{T}=\left[\begin{array}{llll}A & B & C & D\end{array}\right]$ and $p^{T}=\left[\begin{array}{llll}x & y & z & 1\end{array}\right]$. Thus, the intersection with the line $\Gamma_{i, j}$ is given by

$$
t=\Omega^{T} p / \Omega^{\prime T} q_{i, j}
$$

where $\Omega^{\prime T}=\left[\begin{array}{lll}A & B & C\end{array}\right]$ are the reduced co-ordinates of the plane. 
In order to determine the visibility, we look for an intersection with the building's facets in each new grid element in the trajectory. This is performed by evaluating equation (3) for the planes defined by the facets of the buildings stored in the current grid element. If an intersection with a facet is found, then the points are not visible and the search for the grid elements defining the line trajectory is stopped. If the facets of the building in the grid element do not produce an intersection, then we verify for other grid elements in the trajectory until the grid element containing the point $p_{j}$ is reached. If we reach the last element in the trajectory, then we consider that the points are visible. The example in Figure 4(b) shows the case when an intersection is found in the trajectory. Figure 6 shows two examples of the results obtained with the visibility computation process. In this figure, we highlight the facets and edges that are visible from a transmitter position. Figure 6(a) shows the visible facets and figure 6(b) shows the visible edges.

\section{Radiance model}

In a deterministic model, predictions are obtained by modelling the energy transfers. However, energy can be represented by alternative physical measurements such as power, irradiance, radian exposition, radiosity or radiance. These radiometric definitions can be used to express the energy emitted or travelling through space and they can be related to reflection, diffraction, incidence and emittance phenomena. Radiant intensity measures the power that is leaving an area in a particular direction $(\theta, \varphi)$. For an isotropic power, intensity is independent of the outgoing direction and it is obtained by dividing the power by $4 \pi$. If the power $P$ radiates non-isotropic, in one hemisphere then

$$
I(\theta, \varphi)=P G(\theta, \varphi) / 2 \pi
$$

for $G(\theta, \varphi)$ a normalised dimensionless factor. This measure is very useful to model received and transmitted power from point sources. However, to model the incident and reflected 
energy on a surface, we need to account for surface orientation and area. The radiance of a surface is the power per unit of foreshortened area of the source (projected area) per solid angle. If a surface has an area $A$, then the radiance is

$$
L(\theta, \varphi)=I(\theta, \varphi) / A \cos (\theta)
$$

Figure 7 illustrates the concept of radiant intensity and radiance for a $2 \mathrm{D}$ slice of the hemisphere. The term $A \cos (\theta)$ defines an approximate value of the projection of $A$ into the hemisphere. This is only and approximation since the projected area is computed as a tangent plane. Thus, accurate modelling requires areas to be small.

Radiosity defines the radiant power emitted into the hemisphere per unit of area. That is,

$$
B=\int_{\Omega} I(\theta, \varphi) d \omega
$$

This measure is independent of direction. For diffuse surfaces, radiance is given by $L=L(\theta, \varphi)$. Thus, $B=L \pi$. The radiosity equation [24] uses this uniform dispersion property to express the radiosity of a set of surfaces as the sum of the emitted and reflected radiosity of the other surfaces [14][15][25]. As such the power in each surface can be obtained by minimising a system of equations whose solution represents an equilibrium. That is, a point where the reflected power is equal to the incident power plus the constant emitance. Here we consider the energy in terms of the radiance. Radiance maintains the directional dependence and can include specular and diffuse components by modelling reflections by a reflectance function. Additionally, it can be used to model diffractions and fading due to multiple propagations. Our model can be described by two main components: (i) geometric relationships and (ii) energy transfers. The first component is defined by transfer links and the second component by means of an equilibrium equation. 


\subsection{Transfer Links}

The propagation between facets is performed by defining links that represent the transfer of energy from one surface to the other. In general, it is impossible to accurately model energy transfers by considering transfers between the whole surfaces; it is necessary to consider small elements for which the geometry of the transfer can be correctly approximated. As such, for each pair of visible surfaces, we create a set of links by a recursive subdivision process that stops when the error in the geometric approximation (the unoccluded form factor in equation 17) is lower than a fixed threshold. The recursion maintains a hierarchy of sub-facets representing different levels. Links are established only if surfaces are visible according to two cases. First, when there is a direct line-of-sight between the facets. Secondly, when two facets do not have direct line-of-sight, but they have a direct line-of-sight to an edge. This last case includes multiple diffractions. That is, when there is a succession of edges that maintain a direct line-of-sight. Figure 8 illustrates two examples of links with reflection and diffraction effects. The link between the small patches A and B models reflection. The link between $\mathrm{A}$ and $\mathrm{C}$ models a single diffraction.

We denote the value of a link from the facet $i$ to the facet $j$ as $H_{i, j}$. This value is used to define the radiance transfer equation [26][27]

$$
L_{i}\left(\theta_{o}, \varphi_{o}\right)=\sum_{j} H_{i, j} L_{j}(\theta, \varphi)
$$

where $L_{j}(\theta, \varphi)$ denotes the radiance of the $j^{\text {th }}$ facet in an direction $(\theta, \varphi)$ and $L_{i}\left(\theta_{o}, \varphi_{o}\right)$ the radiance reflected by the $i^{\text {th }}$ facet. Equation (7) provides a model of the power transport between surfaces. Variables may be either radiance intensity [28] or radiant power [29]. We use this equation to provide a simplified model of the power transport between two surfaces. In our model, the factor $H_{i, j}$ is defined by 


$$
H_{i, j}=D_{i, j} F_{i, j} G_{i, j}
$$

These components model the diffraction, the power transfer and the phase fading, respectively.

The value $D_{i, j}$ in equation (8) represents the multiplicative loss due to diffraction. In general, the diffraction losses can be computed by considering any multiple edge diffraction method. In the results presented in this paper, the loss is determined based on the Deygout method [1]. This method provides accurate results for few edges with few computations. The diffraction parameter

$$
v_{i, j}=2 \sqrt{\left(\left(d_{i, k}+d_{k, j}\right)-d_{i, j}\right) / \lambda}
$$

is used to determine the loss. In equation (9), $d$ denotes the distance between the facets and the edge.

The third factor for the link in equation (8) represents the direct path loss and it includes attenuation due to reflectivity and geometry. In order to determine this path contribution factor, we consider pair-wise exchanges between facets, the transmitter and the receiver. This factor is explained in sections 5.2 and 5.3. Section 5.4 explains the phase fading factor.

\subsection{Direct Path Loss between Two Facets}

Figure 9(a) illustrates the geometry between two facets. We are interested in obtaining the outgoing radiance $L_{i}\left(\theta_{o}, \varphi_{o}\right)$. First, we determine the incident power in the $i^{\text {th }}$ facet. Since radiance is given in unit of projected area, then the radiated intensity for an infinitesimal area $\Delta_{j}$ is given by

$$
I_{j}(\theta, \varphi)=L_{j}(\theta, \varphi) \cos \left(\theta_{j, i}\right) \Delta_{j}
$$


where $\theta_{j, i}$ is the angle between the surface normal and the straight line between the surfaces. The value of $I_{j}(\theta, \varphi)$ is the power delivered toward the $i^{\text {th }}$ facet. Only a fraction of this power reaches the $i^{\text {th }}$ facet. This fraction can be determined by the angular aperture obtained by projecting an infinitesimal area $\Delta_{i}$. That is,

$$
\Phi=\cos \left(\theta_{i, j}\right) \Delta i / d_{i, j}^{2}
$$

Thus, the incident power is

$$
I_{i}(\theta, \varphi)=L_{j}(\theta, \varphi) \frac{\cos \left(\theta_{j, i}\right) \cos \left(\theta_{i, j}\right)}{d_{i, j}^{2}} \Delta_{i} \Delta_{j}
$$

The power reflected by the surface depends on its reflectance properties. We denote as $\rho_{i}\left(\theta, \varphi, \theta_{o}, \varphi_{o}\right)$ the bidirectional reflectance of the surface. This is expressed in inverse steradian units $\left(s r^{-1}\right)$. Notice that in radiosity models the reflectance is given as the ratio of reflected power defined as the unidimensional reflectance constant $\pi \rho_{i}$ [Fournier95]. This is because for Lambertian reflectors the reflectance is independent of the incidence and reflectance directions. In our model, we consider the more general definition

$$
\rho_{i}\left(\theta, \varphi, \theta_{o}, \varphi_{o}\right)=L_{i}\left(\theta_{o}, \varphi_{o}\right) / E_{i}(\theta, \varphi)
$$

where the irradiance $E_{i}(\theta, \varphi)=I_{i}(\theta, \varphi) / A_{i}$ defines the incident power per unit of area and $A_{i}$ represents the area of the $i^{\text {th }}$ facet. Thus, equation (12) can be rewritten to express the relationship between the radiance of the facets as

$$
L_{i}(\theta, \varphi)=L_{j}(\theta, \varphi) \rho_{i}\left(\theta, \varphi, \theta_{o}, \varphi_{o}\right) \frac{\cos \left(\theta_{j, i}\right) \cos \left(\theta_{i, j}\right)}{A_{i} d_{i, j}^{2}} \Delta_{i} \Delta_{j}
$$

The bidirectional reflectance function models the directional dependence of the transmission. We model reflection by the isotropic component in the Phong model [31]. That is, 


$$
\rho_{i}\left(\theta, \varphi, \theta_{o}, \varphi_{o}\right)=a_{i} \cos ^{n}(\alpha)
$$

where $\alpha$ is the angle between the perfect mirror outgoing vector and the outgoing vector. The parameter $a_{i}$ models absorption and the specular-reflection exponent $n$ controls the aperture of the reflection. High values of $n$ model sharp focused reflections and $n=0$ for perfect diffuse surfaces. Thus, the bidirectional response in equation (14) is

$$
F_{i, j}(\theta, \varphi)=a_{i} \frac{\cos ^{n}\left(\alpha_{i, j}\right) \cos \left(\theta_{j, i}\right) \cos \left(\theta_{i, j}\right)}{A_{i} d_{i, j}^{2}} \Delta_{i} \Delta_{j}
$$

For finite areas, we consider that the energy incident on the surface of the $i^{\text {th }}$ facet is the sum of the energy at each point. However, each point receives power for each point in the $j^{\text {th }}$ facet. Thus, since radiance is measured by unit of area, we have that

$$
F_{i, j}(\theta, \varphi)=\int_{\Delta_{i} \Delta_{j}} a_{i} \frac{\cos ^{n}\left(\alpha_{i, j}\right) \cos \left(\theta_{j, i}\right) \cos \left(\theta_{i, j}\right)}{A_{i}^{2} d_{i, j}^{2}} d_{i} d_{j}
$$

\subsection{Path loss for an Antenna and a Facet}

In the previous section, we considered the emitted radiance of a facet due to the variance of another facet. In this section we shall consider the relationship between the radiance of a facet and the receiver and transmitter power. In radiosity techniques, it is possible to multiply radiosity by area, so the transfer equation can be expressed in terms of total power emitted into the hemisphere [18][32]. However, power is expressed as a uniform dispersion. In order to keep power dependent on the ingoing and outgoing directions we consider two propagation phenomena. First, we use the concept of radiance intensity to determine the power incident on the facet. Secondly, we use the reflectance and the geometry of the facet to obtain the emitted radiance by following the plane surface model [20]. Figure 8(b) illustrates the geometry between a transmitter and a facet. We are interested in obtaining the outgoing radiance $L_{i}\left(\theta_{o}, \varphi_{o}\right)$ given the transmitter power $P_{a}(\theta, \varphi)$. 
The radiant intensity of a transmitter with a radiant power $P_{a}$ and gain $G_{a}(\theta, \varphi)$ is given by

$$
I_{a}(\theta, \varphi)=P_{a} G_{a}(\theta, \varphi) / 4 \pi
$$

This defines the power in the direction $(\theta, \varphi)$ per steradian. The well-known free-space equation

$$
I_{a}(\theta, \varphi)=P_{a} G_{a}(\theta, \varphi) G_{r} \lambda^{2} / 4 \pi d^{2}
$$

can be obtained by considering a receiver at distance $d$ with an effective area $A_{a} \approx \lambda^{2} / 4 \pi$ and gain $G_{r}$. However, we do not have a receiver at the other end, but we are interested in obtaining the power incident on a small flat area. This power depends on the distance, the area and the surface orientation. The angular aperture of a surface at a distance $d_{i a}$ with a small area $\Delta_{i}$ and with an angle $\theta_{i a}$ (Figure 9(b)) is given by

$$
\Phi=\cos \left(\theta_{i, a}\right) \Delta i / d_{i, a}^{2}
$$

Thus the incident power is

$$
I_{i}(\theta, \varphi)=P_{a} G_{a}(\theta, \varphi) \frac{\cos \left(\theta_{i, a}\right)}{4 \pi d_{i, a}^{2}} \Delta_{i}
$$

Once we know the incident power, we can obtain the emitted radiance by considering the reflected angle geometry of the facet. By considering equation (13) we have that the radiance of the facet is

$$
L_{i}(\theta, \varphi)=P_{a} G_{a}(\theta, \varphi) \rho_{i}\left(\theta, \varphi, \theta_{o}, \varphi_{o}\right) \frac{\cos \left(\theta_{i, a}\right)}{4 \pi d_{i, a}^{2}} \Delta_{i}
$$

If the bidirectional reflectance function is given by equation (15), then we have

$$
F_{i, a}(\theta, \varphi)=a_{i} \frac{\cos ^{n}\left(\alpha_{i, a}\right) \cos \left(\theta_{i, a}\right)}{4 \pi d_{i, a}^{2}} \Delta_{i}
$$


For a finite area, we must consider that the energy incident on the surface of the $i^{\text {th }}$ facet is the sum of the energy at each point. Thus,

$$
F_{i, a}(\theta, \varphi)=\int_{\Delta_{i}} a_{i} \frac{\cos ^{n}\left(\alpha_{i, a}\right) \cos \left(\theta_{i, a}\right)}{4 \pi A_{i} d_{i, j}^{2}} d_{i}
$$

To model the power at a receiver, we compute the incident power from a facet and then we multiply it by the effective area of the receiver. The radiated intensity of a facet for an infinite area is given in equation (10). The available power for a receiver at a distance $d_{i, r}$ is

$$
I_{r}(\theta, \varphi)=G_{r} L_{i}(\theta, \varphi) \frac{\lambda^{2} \cos \left(\theta_{i, r}\right)}{4 \pi d_{i, r}^{2}} \Delta_{i}
$$

Here, the factor $\lambda^{2} / 4 \pi d_{i, r}^{2}$ defines the effective area, and $G_{r}$ is the gain of the receiver antenna. For a finite facet area we have

$$
I_{r}(\theta, \varphi)=\frac{\lambda^{2} G_{r}}{4 \pi} \int_{A_{j}} L_{j}(\theta, \varphi) \frac{\cos \left(\theta_{j, i}\right)}{d_{i, j}^{2}} d_{j}
$$

\subsection{Phase Shift}

In order to include channel fading, equation (4) can be interpreted by considering inphase and quadrature-phase components. These components are determined based on the dominant component incident on each facet. That is, if the angle of the dominant phasor of a facet $j$ is $\gamma_{j}$, the arriving wave at facet $i$ experiences a phase shift of

$$
\gamma_{j}+\alpha_{j, i}
$$

where $\alpha_{j, i}$ is the shift in a distance $d_{i, j}$. Thus, in order to compute the fading interference, we define the last factor in equation (5) as

$$
G_{i, j}=e^{\left(\gamma_{j}+\alpha_{j, i}\right)}
$$

Thus, equation (4), actually defines a vector sum. In practice, we use the modulus 


$$
L_{i}\left(\theta_{o}, \varphi_{o}\right)=\left\|\sum_{j} H_{i, j} L_{j}(\theta, \varphi)\right\|
$$

to obtain the transfer power and we consider $\gamma_{i}$ to be the phase shift for the incident link with the strongest power.

\subsection{Transfer Equation}

Equation (29) models the energy transfer from a surface to another. The unknowns of this equation are the radiances $L_{i}$ and $L_{j}$. Similarly to the radiosity equation [24] this equation defines a system where each row $i$ determines the radiance as the weighted sum of the radiance of other surfaces. However, it represents the reflected energy as a function rather than as single value. Reflected energy functions have been studied in [33] and [30]. In our implementation, the solution is obtained by considering discrete values of the functions $L_{i}\left(\theta_{o}, \varphi_{o}\right)$ and $L_{j}\left(\theta_{o}, \varphi_{o}\right)$. The discrete values $L_{i, \theta_{o}, \varphi_{o}}$ define the radiance in a particular direction. Thus,

$$
L_{i, \theta_{o}, \varphi_{o}}=\left\|\sum_{j} H_{i, j} L_{j, \theta, \varphi}\right\|
$$

In order to find the solution, we first use equation (22) to compute the radiances for facets that have a link to the transmitter. This gives to some facets a constant emittance that provide an initial solution. When the equilibrium solution is found, then the power to the receiver can be computed by considering the links to the receiver according to equation (26) and the direct path to the transmitter.

In order to find a solution it is necessary to gather incoming power for each facet. In our implementation is based on the hierarchical approach [15][21]. In this approach, the radiance of each surface is gathered and updated by traversing the hierarchical subdivision. Each part in the subdivision represents a new reflector that is linked to other facets. The gathering is 
performed by following the links originating at each facet. Values are efficiently and consistently maintained by a bi-directional traversal of the hierarchy. In the first traversal, accurate values are obtained at the lowest level (i.e., small subdivision) by adding the contributions of each subdivided facet. That is, starting from the top of the facet hierarchy, the power of the sub-element is just added to each of its descendants to push down correct power values. Once the push process is finished, then in a second traversal, all the values in the hierarchy are updated according to the accurate values at the bottom. Thus, power values are pulled up. Since we are modelling power per unit of area, then the correct values are given by the area weighted average.

\section{Results}

The model was used to predict path-loss for two transmitter positions. We use a single value of $a_{i}$ and $n$, in equation (15), for all buildings $\left(a_{i}=0.86, n=18\right)$. These values were obtained by minimising the prediction error. Figure 10 shows the prediction results obtained for the transmitter located at Karstadt. Since at the border of the city there are not buildings that reflect the signal back, then shadows are produced. However, we can see how the reflection and diffraction make accessible the signal in the corridors formed by buildings. The image shows a congruent prediction with the data base configuration. To validate the results, we compare the prediction against field measurements. The graphs in figures 10 and 11 show the measurements, predictions, error and correlation for the two transmitters located in Karstadt and Universitaet, respectively. For Karstadt we used 1420 field measurements and for Universitaet we used 2140 field measurements. We can observe that there is a good agreement for most locations. This is confirmed by the statistics shown in Table 1 . The correlation coefficient and the mean error show that the predictions follow the major trends. Similar results were obtained for both transmitters showing a good agreement of the model. 
Differences between measurements and predictions are due to three main factors. First, there are some predictions with infinite path loss shown as zero values in the graphs. This is mainly due to lack of information in the database. For these points the contribution comes from a building that it is not present in the database, as such reflection is not obtained by the model and the point prediction does not have a value. This is a typical case for the receivers near the limit of the database (i.e., the dark regions in the border of Figure 9). A second source of error is due to the limited number of reflections in the model. In some cases the signal power can be significant after several reflections. However, computations limit the possible number of interactions. The results presented in this paper where obtained by considering six order reflections. A better strategy should adaptively control the number of reflections and diffractions to minimise computation maximising energy transfers. Finally, a third source of error comes from the fact that we consider that all buildings have the same reflective properties. In general, the absorption and aperture of reflections are not homogeneous for all the buildings. Thus, these parameters could be set for different areas of the database. However, this can be computationally complex.

\section{Conclusions}

We have implemented a model of path-loss prediction based on global facet-to-facet radiance transfers. The model is based on radiosity techniques and it considers the visibility geometry of the power transfer between surfaces. The main differences between our model and radiosity techniques are: (i) our model considers diffraction, (ii) it models specular and diffuse scattering and (iii) it considers fading effects. Since the diffuse component is neglected, then the equilibrium equation can be solved for just specific directions using iterative methods. Our implementation is based on the hierarchical radiosity algorithm. This handles efficiently facet sub-divisions necessaries to obtain an accurate prediction. Results show a congruent prediction with data measurements capable of providing accurate 
propagation predictions. The main drawback is that it requires a fair estimate of the reflective properties of facets.

\section{References}

[1] C. López Giovaneli, An analysis of simplified solutions for multiple knife-edge diffraction, IEEE Transactions on Antennas and Propagation AP-32(3):297-301 (1984).

[2] J. Walfish, H.L. Bertoni, A theoretical model of UHF propagation in urban environments, IEEE Transactions on Antennas Propagation 38(12):1788-1796 (1988).

[3] IEEE Transactions on Vehicular Technology, Special Issue on Mobile Radio Propagation 37(1), 1988.

[4] G. Liang, H.L. Bertoni, A new approach to 3-D ray tracing for propagation prediction in cities, IEEE Transactions on Antennas and Propagation 46(6):853-863 (1998).

[5] J.W. Ikegami, T. Takeuchi, S. Yoshida, Theoretical prediction of map field strength for urban mobile radio, IEEE Transactions on Antennas and Propagation 39:299-302 (1991).

[6] J.W. McKown, R.L. Hamilton, Ray tracing as a design tool for radio networks, IEEE Network Magazine 5(6):27-30 (1991).

[7] K.R. Schaubach, N.J. Davis, T.S. Rappaport, A ray tracing method for predicting path loss and delay spread in microcellular environments, Proc. IEEE Vehicular Technology Conference, pp. 932-935 (1992).

[8] R.A. Valenzuela, A ray tracing approach to predicting indoor wireless transmission, Proc. IEEE Vehicular Technology, pp. 214-218 (1993).

[9] D.J. Cichon, W. Wiesbeck, Ray optical wave propagation modelling in urban micro cells, PIMRC'94, pp. 407-410 (1994).

[10] V. Erceg, A.J. Rustako, R.S. Roman, Diffraction around corners and its effects on the microcell coverage area in urban and suburban environments at $900 \mathrm{MHz}, 2 \mathrm{GHz}$, and 7GHz, IEEE Transactions on Vehicular Technology 43:762-766 (1994).

[11] B.E. Gschwendtner, G. Wölfle, F.M. Landsforfer, Ray tracing vs, ray launching in 3-D microcell modelling, Proc. European Personal and Mobile Communications Conference, EPMCC'95, pp. 74-79 (1995).

[12] J.P. Rossi, J.C. Bic, A.J. Levy, A ray launching method for radio - mobile propagation in urban area, Proc. IEEE Antennas and Propagation Symposium, pp. 1540-1543 (1991).

[13] S.Y. Tan, H.S. Tan, Propagation model for microcellular communications applied to path loss measurements in Ottawa city streets, IEEE Transactions on Vehicular Technology 44(2):313-317 (1995).

[14] C.M. Goral, K.E. Torrance, D.P. Greenberg, B. Battaile, Modeling the interaction of light between diffuse surfaces, Computer Graphics 18: 213-222 (SIGGRAPH'84 Proceedings) (1984).

[15] F.X. Sillion, C. Puech, Radiosity and global illumination, Morgan Kaufmann Publishers, San Francisco California (1994). 
[16] C. Kloch, J.B. Andersen, Radiosity - An approach to determine the effect of rough surface scattering in mobile scenarios, Proc. IEEE Antennas and Propagation Society Int. Symp., Montreal, Canada, pp. 890-893 (1997).

[17] K.-F. Tsang, W.-S. Chan, D. Jing, K. Kang,S.-Y. Yuen, W.-X. Zhang, Radiosity Method: A new propagation model for microcellular communication, Proc. IEEE Antennas and Propagation Society Int. Symp. 4:2228-2231 (1998).

[18] C. Kloch, G. Liang, J.B. Andersen, G.F. Pedersen, H.L. Bertoni, Comparison of measured and predicted time dispersion and direction of arrival for multipath in a small cell environment, IEEE Transactions on Antennas and Propagation 49(9):12541263 (2001).

[19] L. Neumann, A. Neumann, Radiosity and hybrid methods, ACM Transactions on Graphics 14(3):233-265 (1995).

[20] J. D. Parsons, The Mobile Radio propagation Channel (Wiley \& Sons, 1992)

[21] P. Hanrahan, D. Salzman, L. Aupperle, A rapid hierarchical radiosity algorithm, Computer Graphics 25(4):197-206, (SIGGRAPH'91 Proceedings) (1991).

[22] N. Dadoun, D. Kirkpatrick, and J. Walsh, The Geometry of Beam Tracing, Proc. ACM Symposium on Computational Geometry, pp. 55-61 (1985).

[23] S. Coorg, S. Teller, Temporally coherent conservative visibility, Proc. $12^{\text {th }}$ Symp. Computational Geometry, ACM, pp. 78-87 (1996).

[24] J.T. Kajiya, The rendering equation, Computer Graphics 20(4):143-150, (SIGGRAPH'86 Proceedings) (1986).

[25] T. Nishita, E. Nakamai, Continuous tone representation of three-dimensional objects taking account of shadows and interreflection, Computer Graphics 18:23-30, (SIGGRAPH'84 Proceedings) (1984).

[26] R. Siegel, J.R. Howell, Thermal radiation heat transfer, Mc Graw Hill, New York 1981.

[27] M.F. Cohen, J.R. Wallace, Radiosity and realistic image synthesis, Academic Press (1993).

[28] D.S. Immel, M.F. Cohen, D.P. Greenberg, A radiosity method for non-diffuse environments, Computer Graphics 20(4):133-142, (SIGGRAPH'86 Proceedings), (1986).

[29] L. Neumann, A. Neumann, Photosimulation: interreflection with arbitrary reflectance models and illumination, Computer Graphics Forum 8(1):21-34 (1984).

[30] A. Fournier, Separating reflection functions for linear radiosity, Eurographics Workshop on rendering, pp. 296-305, (1995).

[31] R. Lewis, Making shaders more physically plausible, Eurographics'94 13(3):1-13 (1994).

[32] M.Z. Shao, O.S. Peng, Y.D., Liang, A new radiosity approach by perocedural refinements for realistic image synthesis, Computer Graphics 22(4):93-101, (SIGGRAPH'88 Proceedings), (1988).

[33] L. Neumann, A. Neumann, Efficient radiosity methods for non-separable reflectance models, Proc. Eurographics Rendering Workshop on Photosimulation realism and physics in Computer Graphics, pp. 83-102, (1990). 


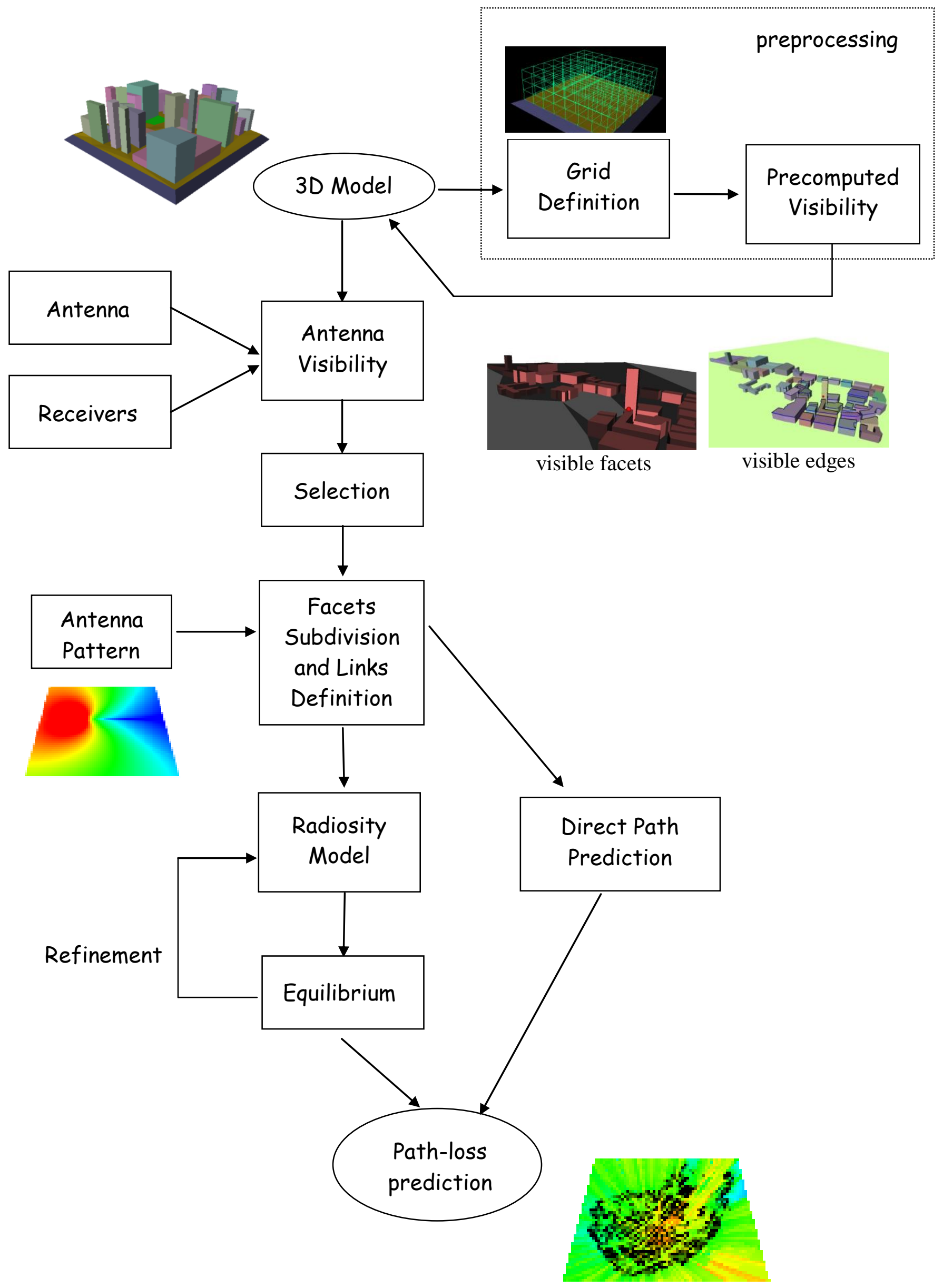

Figure 1. Global radiosity framework. 


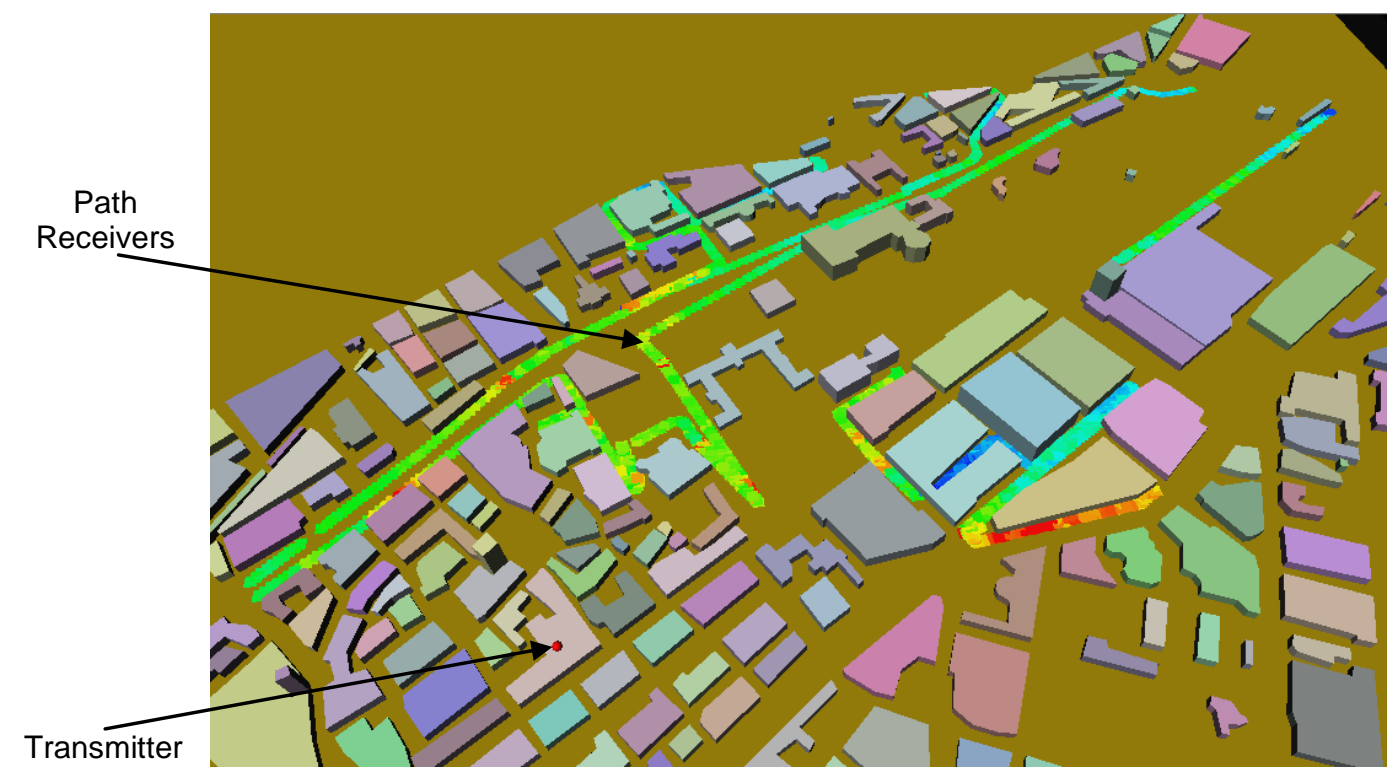

Figure 2. City data with one antenna and a path of receivers.

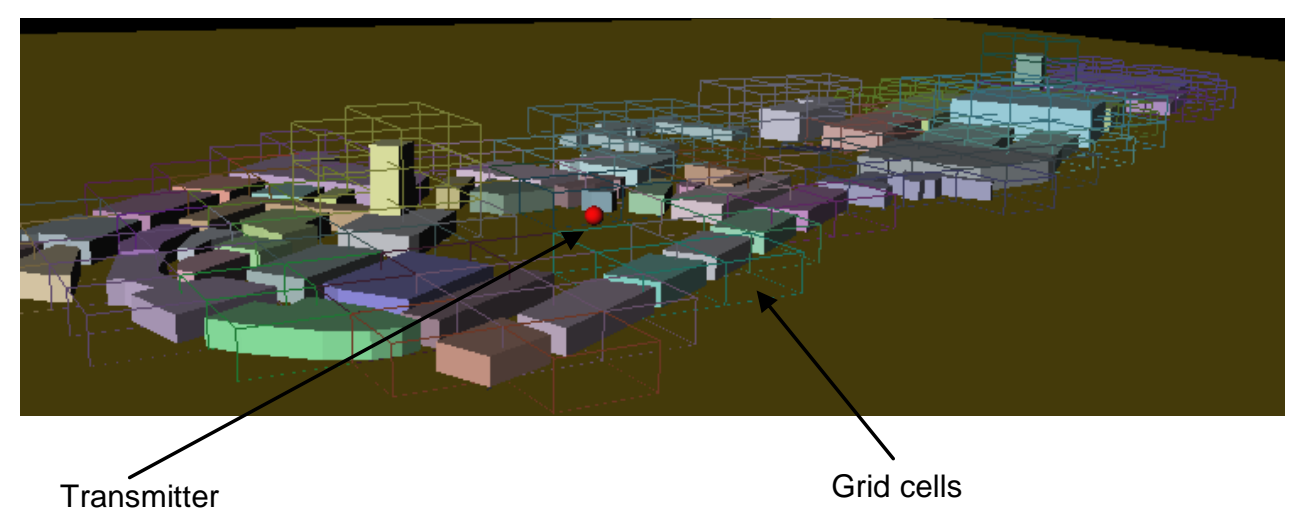

Figure 3. Example of $3 \mathrm{D}$ partition used during visibility computations. 


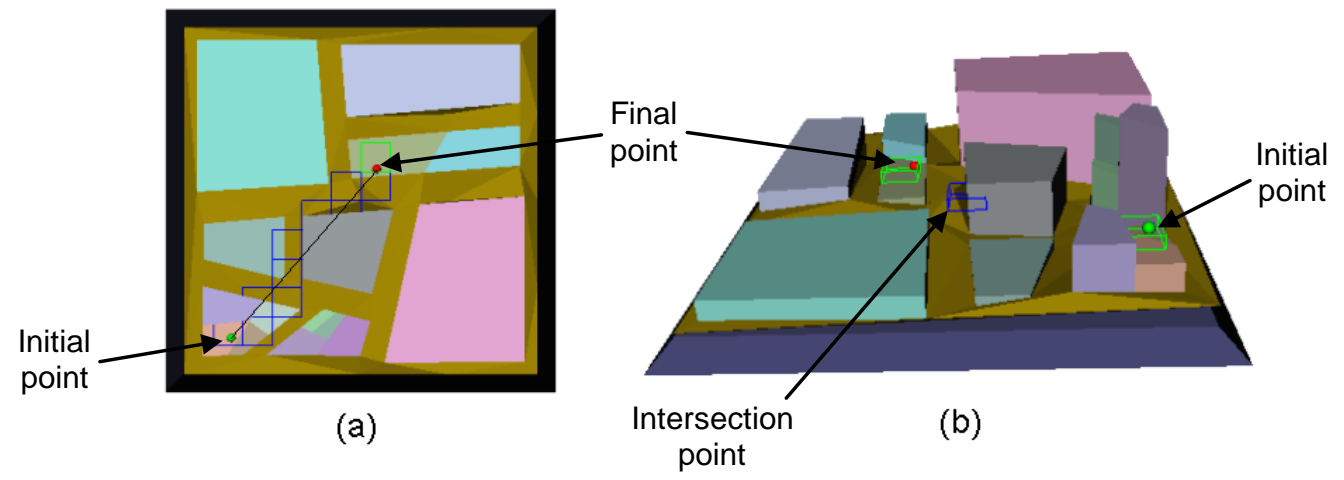

Figure 4. Example of grid elements selected during the computation of the visibility between two points. (a) Top view of the grid trajectory. (b) 3D grid element for the initial, final and intersection points.

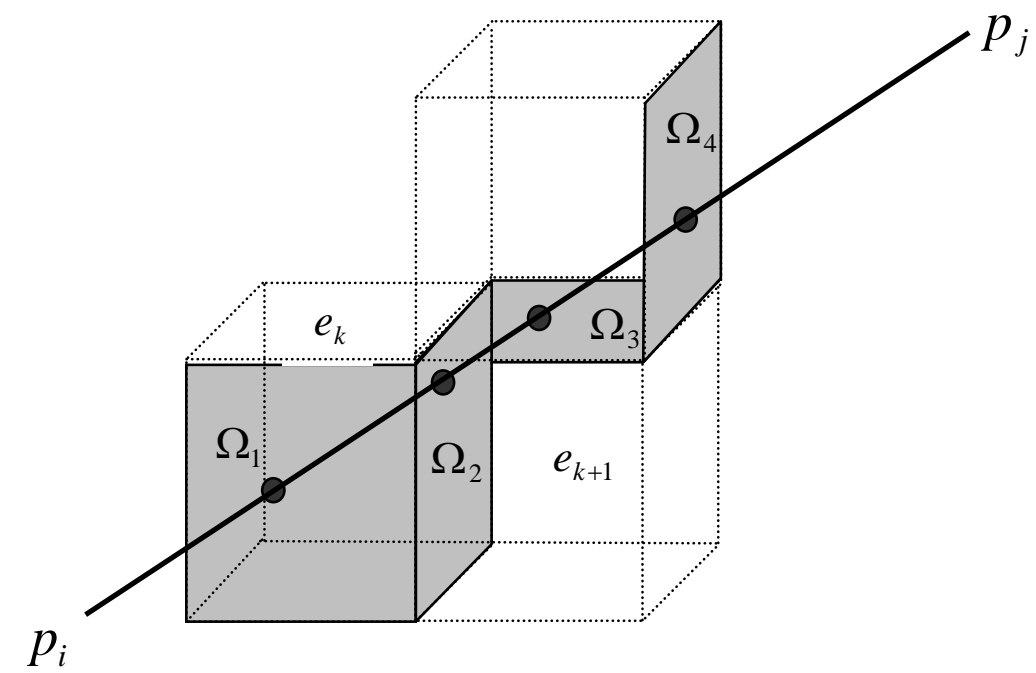

Figure 5. Example of consecutive grid elements. 


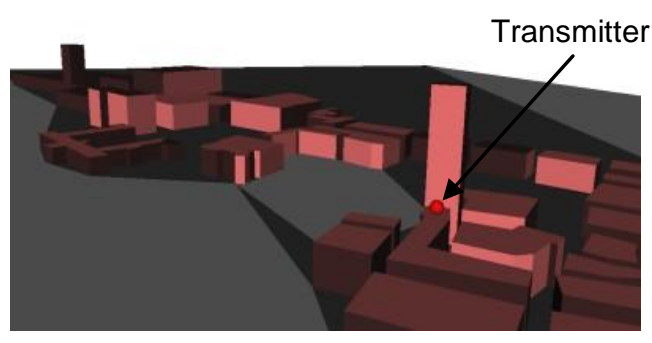

(a)

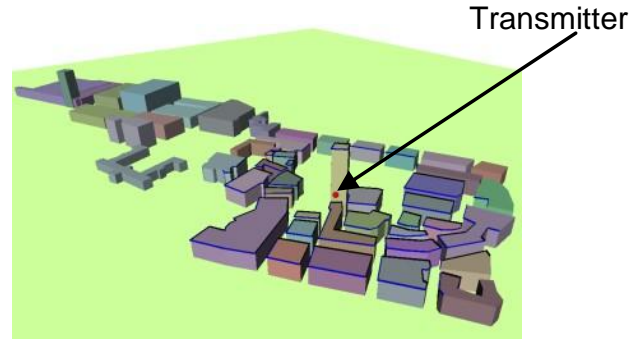

(b)

Figure 6. Visibility results. (a) Example of visible facets. (b) Example of visible edges.

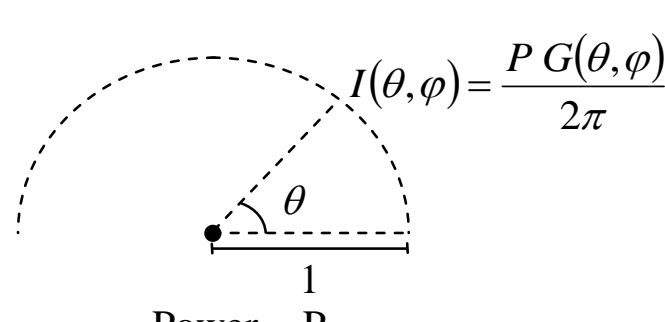

Power $=\mathrm{P}$

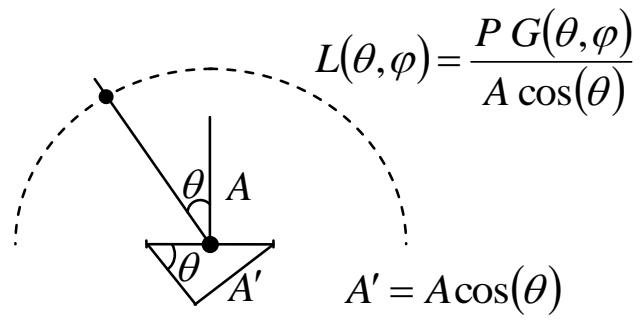

Power $=\mathrm{P}$

Area $=\mathrm{A}$

(a)

(b)

Figure 7. Radiant intensity and radiance of a surface. (a) Radiant intensity gives the power leaving a point. (b) Radiance ..... for surface orientation.

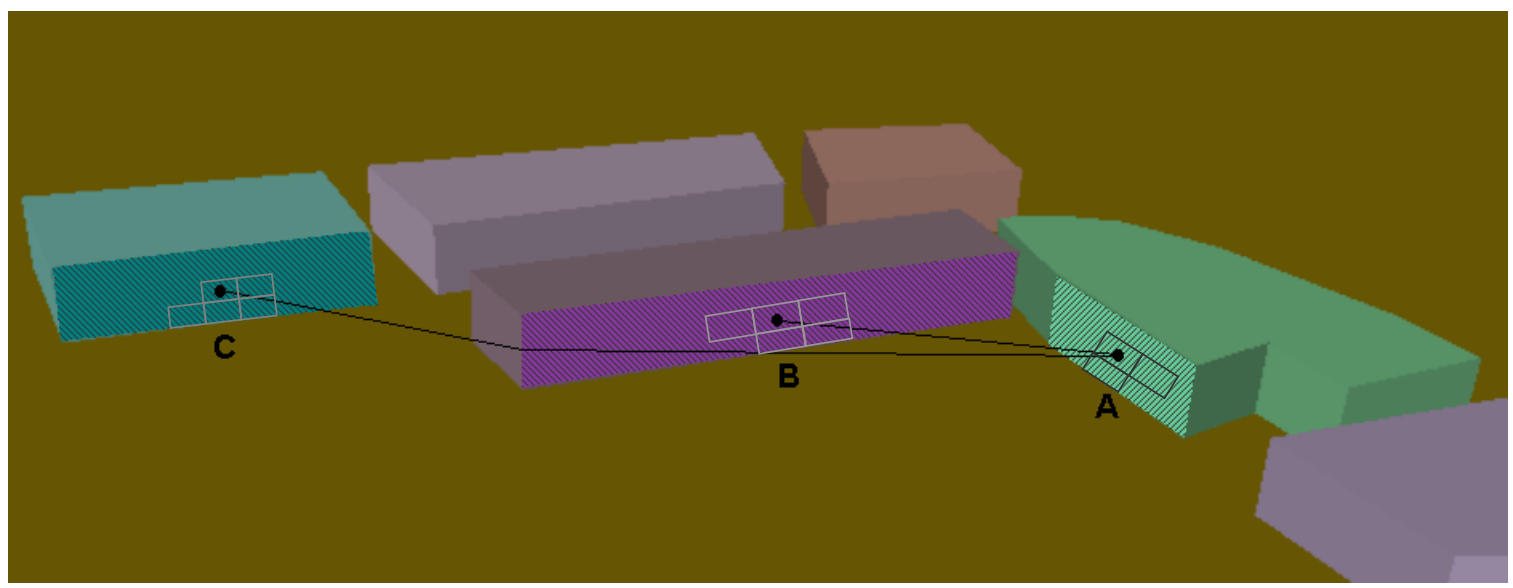

Figure 8. Example of transfer links. The link between A and B models reflection and the link between $\mathrm{A}$ and $\mathrm{C}$ models a single diffraction. 


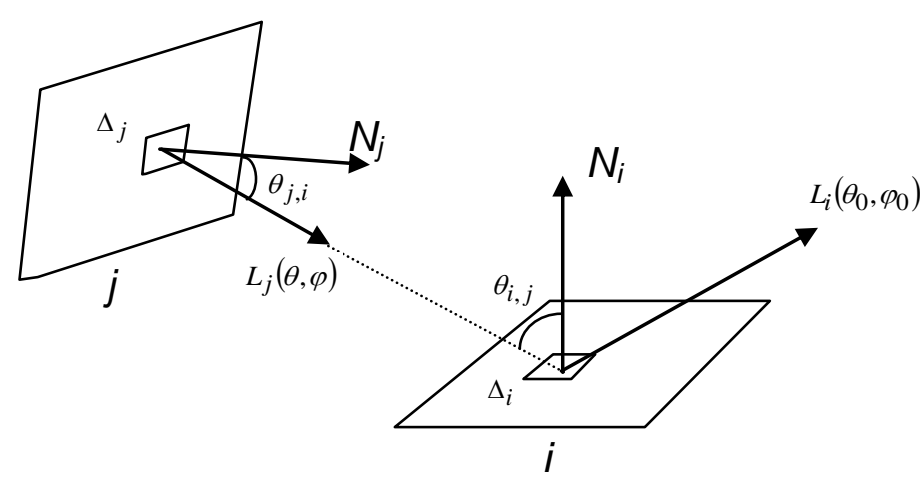

(a)

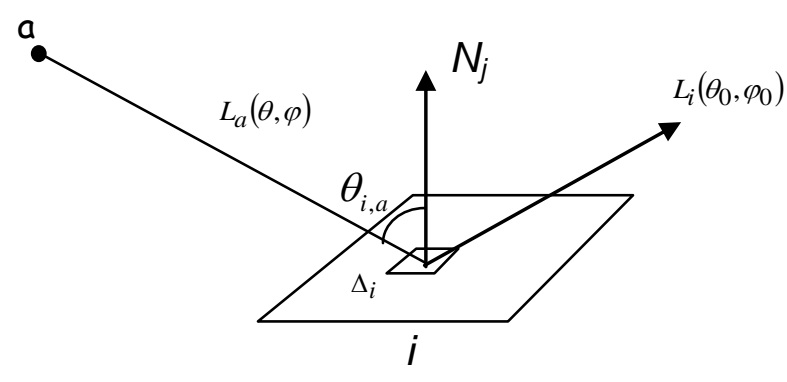

(b)

Figure 9. Geometry of radiance transport. (a) For two facets. (b) For the antenna and a facet.

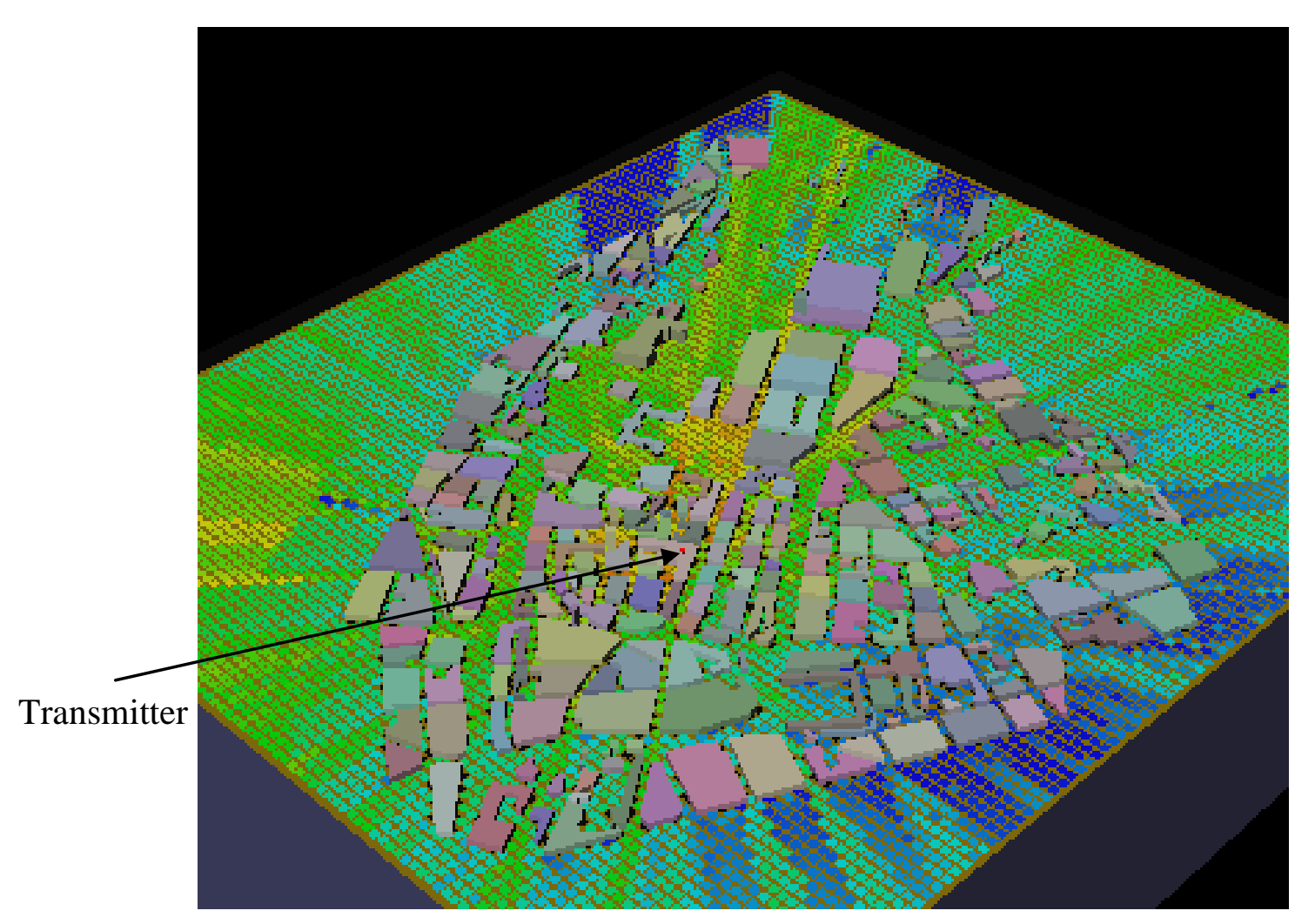

Figure 10. Result of path-loss prediction 


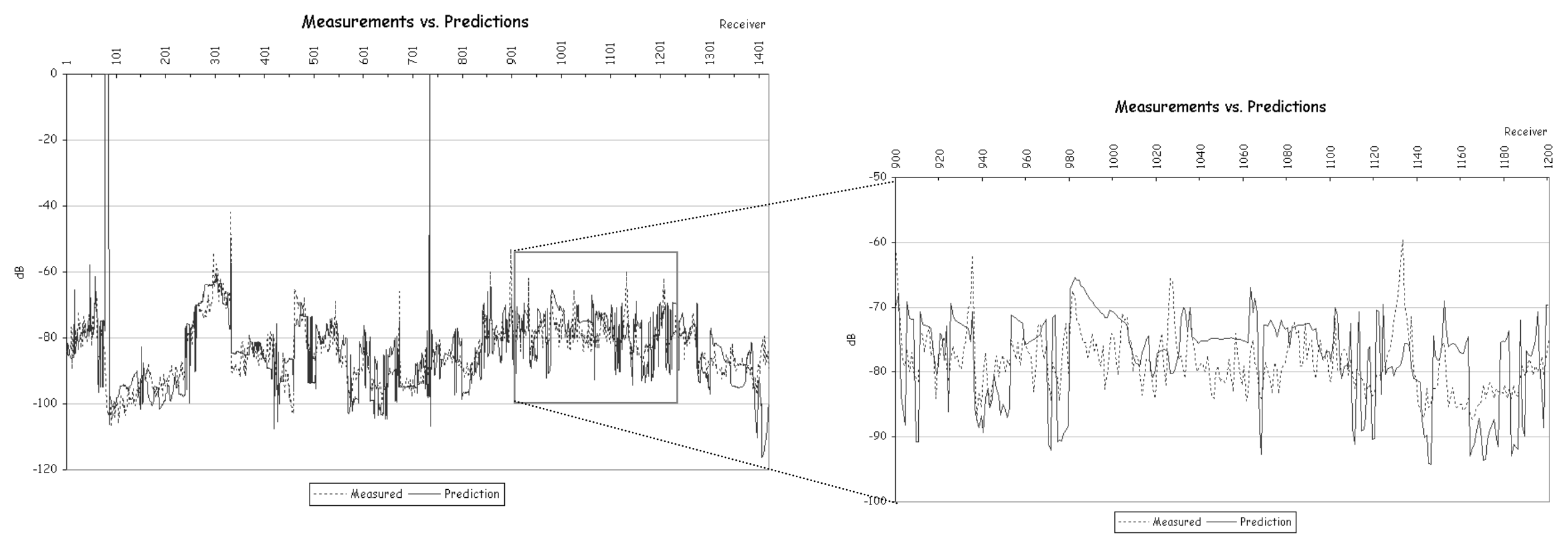

(a)

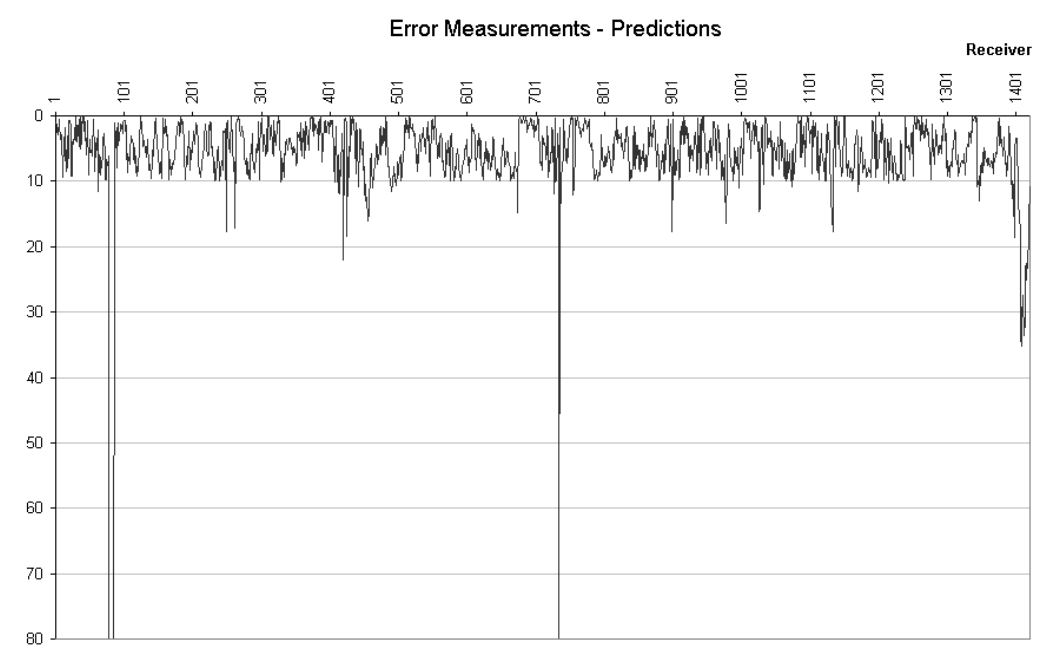

(c) (b)

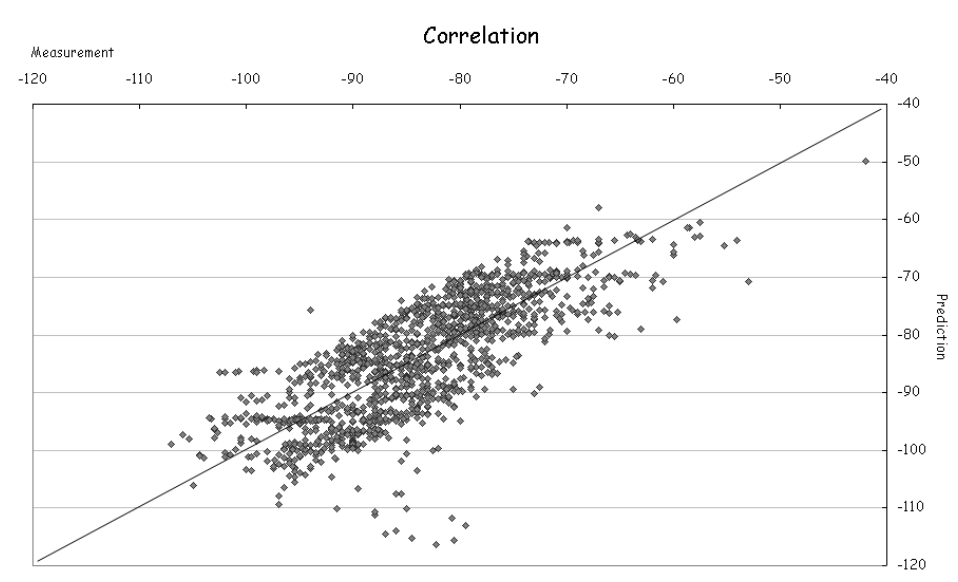

(d)

Figure 10. Comparison of predicted values versus measurements for the transmitter located at Karstadt. (a) Measurements vs. Predictions. (b) Detail of Measurements vs. Predictions. (c) Error between measurements and predictions. (d) Correlation between Measurements and Predictions. 
Measurements vs. Predictions

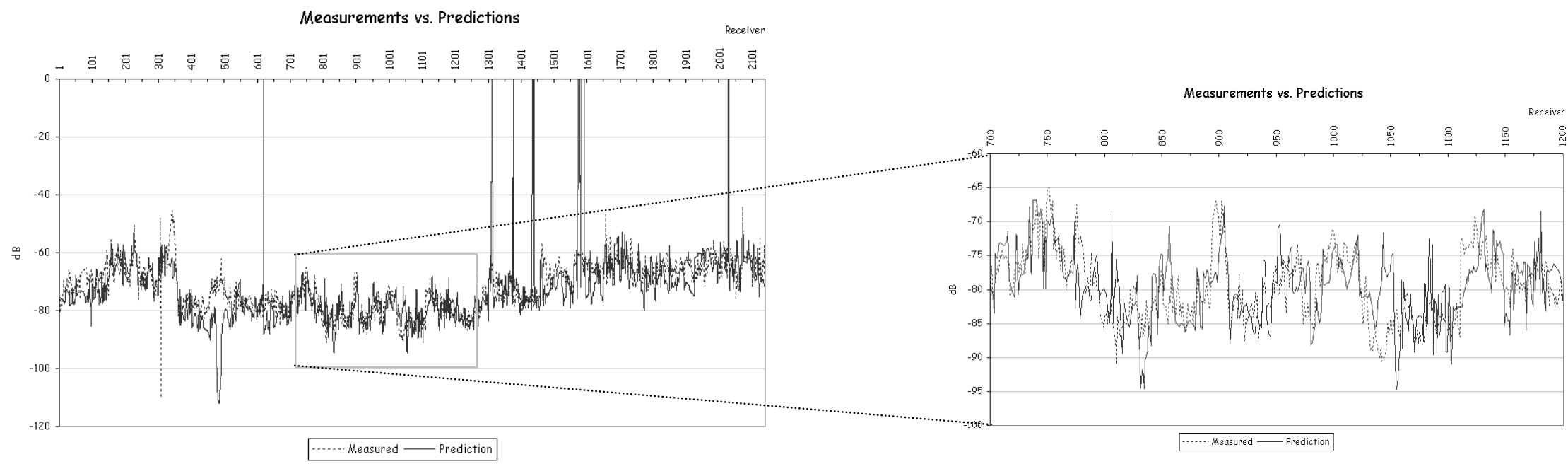

(a)

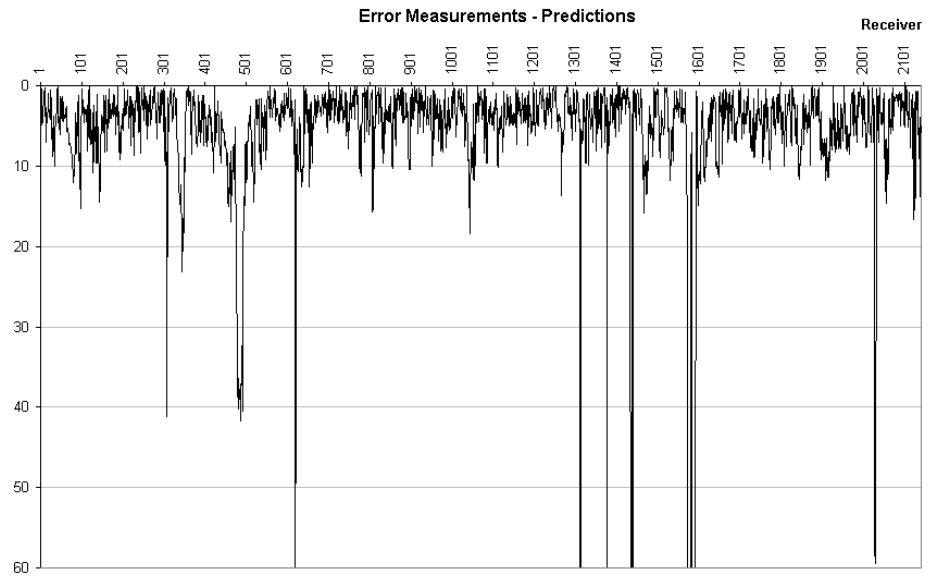

(c) (b)

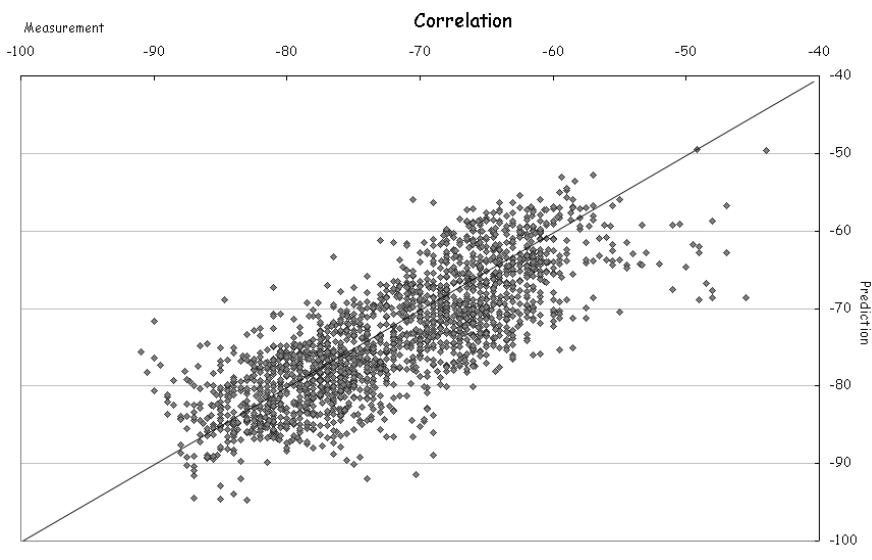

(d)

Figure 11. Comparison of predicted values versus measurements for the transmitter located at Universitaet. (a) Measurements vs. Predictions. (b) Detail of Measurements vs. Predictions. (c) Error between measurements and predictions. (d) Correlation between Measurements and Predictions. 


\begin{tabular}{|l|c|c|}
\hline \multicolumn{1}{|c|}{ Statistics } & Karstadt & Universitaet \\
\hline Mean Error $(\mathrm{dB})$ & 1.77 & 0.22 \\
\hline Standard Deviation Error & 6.61 & 6.64 \\
\hline Correlation & 0.695 & 0.771 \\
\hline
\end{tabular}

Table 1. Statistics obtained by comparing Measurements vs.

Predictions for the two transmitters located in Stuttgart. 